\title{
Enhanced Connectivity and Energy Efficient Networking for Modern Railways Communication Systems
}

\author{
Elias Yaacoub ${ }^{1}$, Ahmad Alsharoa ${ }^{2}$, Hakim Ghazzai $^{3}$, and Mohamed-Slim Alouini ${ }^{4}$ \\ ${ }^{1}$ Dept. of Electrical \&Computer Engineering, American University of Beirut (AUB), Beirut, Lebanon. \\ ${ }^{2}$ Engineering and Computer Science Department, Virginia State University, Petersburg, Virginia, USA. \\ ${ }^{3}$ Stevens Institute of Technology, Hoboken, New Jersey, USA. \\ ${ }^{4}$ Computer, Electrical and Mathematical Sciences and Engineering (CEMSE) Division, King Abdullah University of Science and \\ Technology (KAUST), Thuwal, Makkah Province, Saudi Arabia. \\ Emails:eliasy@ieee.org,aalsharoa@vsu.edu,hghazzai@stevens.edu,slim.alouini@kaust.edu.sa
}

\begin{abstract}
To meet the increasing demands for passenger data rates, modern railway communication networks face significant challenges. The advent of $5 \mathrm{G}$ communications after the long-term evolution (LTE) and LTE-Advanced (LTE-A) systems provides several technological advances to address these challenges. In this paper, after reviewing the main $5 \mathrm{G}$ communication aspects for modern railways, we present methods to ensure connectivity and energy efficiency for the passengers' user equipment (UE) through the use of mobile relays (MRs) on top of the train wagons in conjunction with intelligent resource allocation. Relevant challenges to train connectivity are also presented, and suitable solutions are outlined.
\end{abstract}

\section{INTRODUCTION}

With broadband wireless connectivity expected anytime anywhere, train passengers can no longer be served with the 3G/4G base stations (BSs) of mobile operators deployed to meet the demand of the populated areas in the surroundings of the railroad track. Furthermore, with the increased requirements for efficient train control signaling, real-time transmission of mission-critical data, and accurate monitoring of the railroad status, GSM-R should evolve into the nextgeneration of wireless technologies [1].

Moreover, railroad wireless communications coverage should include BSs dedicated for train passenger connectivity, in addition to, or co-located with, the BSs that are used for the train control network. The BSs can be connected to remote radio heads (RRHs) that are more densely deployed along the track, and communicating with a mobile relay (MR) located on top of the train [2]. An enhanced performance is obtained when two MRs (front and rear) are located on top of the train, with several RRHs per BS deployed along the track [3]. Another approach to increase coverage and capacity is the combination of macro BSs with small cells along the railroad track, with several MRs deployed on top of the train [4].

The role of MRs is essential in enhancing communications. They have outdoor antennas outside the train that are connected to the BS or RRH. With BSs/RRHs deployed along the railroad track, this outdoor connectivity is expected to be line of sight (LOS) most of the time. In addition, these MRs are usually connected to each other by fiber optic cables inside the train, and can thus distribute the received information to the user equipment (UEs) wirelessly using a dedicated network inside the train (e.g., WiFi).

In $[5,6]$, BSs were considered to be deployed along the track to ensure long range (LR) LOS connectivity to MRs, whereas one MR was deployed per wagon (i.e., several MRs per train), with its outdoor antennas connected to the BS and its indoor antennas serving the UEs inside the wagon, where an indoor short range (SR) communication network is formed, as shown in Fig. 1. The SR connectivity can be ensured through WiFi, or through LTE/LTE-A for example.

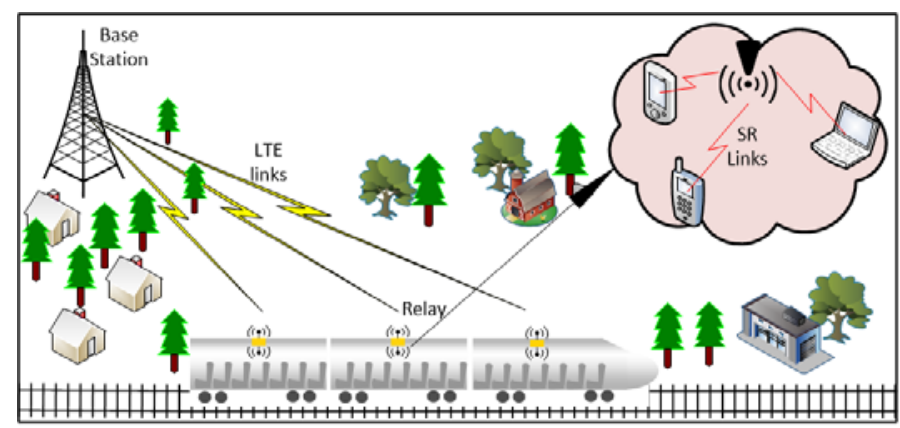

Fig. 1: Railroad connectivity with mobile relays.

This approach saves UEs from suffering large penetration losses in case of direct connection to the BSs, and spares them from being subjected to frequent handovers as the train moves at high speed. An additional benefit is also a reduced power consumption, since it is less energy consuming to obtain the data via SR links with low mobility between UEs and MRs, than over LR links with frequent handovers at high train speed between the UEs and BSs. The scenario shown in Fig. 1 can be enhanced when MRs are connected by fiber, and performance can be boosted by additional RRHs or small cells along the railroad track.

In this paper, we discuss 5G connectivity for railroad, analyzing both the passenger and train control communication 
networks. Then, we present an approach for using MRs in conjunction with intelligent resource allocation, in order to meet the quality of service (QoS) requirements of passengers while minimizing the energy consumption of their UEs. This energy efficient approach also reduces the required transmit power by the MRs and BSs. Challenges related to connectivity in railways are then listed, and appropriate solutions are outlined before concluding the paper.

\section{5G RAILROAD CONNECTIVITY}

A possible approach for designing railway communications consists of using a system for passenger data and another separate system for train control data. This latter system can be even split into two: one for mission critical data and the other for non-mission critical data. However, another approach consists of using one communication system [7]. Indeed, with 5G, a single network can be used for all types of data with appropriate network slicing through software defined networking (SDN) and network function virtualization (NFV) [8]. This approach is preferable, since it allows the allocation of dedicated network resources for each scenario while using a unified and integrated physical architecture, which facilitates network planning, operation, and management.

5G defines three main use cases that all intersect with communications in modern railways:

- Enhanced mobile broadband (eMBB): This use case mainly handles passenger communications. It should cope with the tremendous increase in demand for high data rates due to the use of real-time video streaming, social media, large downloads, etc. It benefits from the presence of MRs to ensure high data rates inside the train while the outdoor antennas of the MRs ensure good connectivity with the BS and/or RRHs. In addition, with 5G, large numbers of antennas can be deployed to form massive multiple-input multiple-output (MIMO) arrays. This is particularly useful for the BS-MR link since it can significantly boost performance. In addition, when MRs are deployed over each wagon as in Fig. 1, they can be controlled by a single baseband unit (BBU) inside the train. Thus, their respective antennas can form a large MIMO array. When each MR is in turn equipped with its own MIMO array, then the joint operation of all the MRs can be identical to that of a large massive MIMO array controlled by the BBU, coupled with LOS with the BS, thus leading to huge communication data rates.

- Massive machine-type communications (mMTC): This use case is dedicated to accommodate a large number of sensing devices accessing the network frequently to send relatively short amounts of data. These could include sensors for monitoring the state of the railroad track, bridges, underground tunnels, etc. Their data can be aggregated locally (e.g., by a controller within the vicinity of a group of sensors) before being sent to the network. The collected data can be processed and analyzed on the servers of the company managing the railways, using data analytics and machine learning, in order to schedule periodic maintenance, predict the expected time before the need for repairs, and perform preventive maintenance, etc.

- Ultra-reliability and low-latency communications (URLLC): This use case corresponds to mission-critical services that do not tolerate delays. For example, it can consist of the transmission of surveillance videos to take appropriate action in case of emergency. Another example consists of realtime control and management data, e.g., sending a command to stop a train due to an emergency, diverting a track into another route, etc. An appropriate $5 G$ network slice should be dedicated to ensuring the required resources for URLLC. Furthermore, if needed in case of emergency, resources from the other slices should be allocated to the URLLC slice as it has the utmost priority.

Another aspect of $5 \mathrm{G}$ communications is the use of various frequency bands, with the use of millimeter wave (mmWave) frequencies being one of the most important 5G features. In [2], an integrated approach is presented where two types of RRHs are used: one type uses the licensed frequencies below $6 \mathrm{GHz}$, and the other type uses mmWave frequencies to meet the increasing capacity of user data. The simulation results of [2] showed a increase in capacity, especially in the vicinity of the RRHs.

However, propagation models for mmWave frequencies at high speed are not well-developed, and there are significant and important research efforts ongoing in this direction, e.g. [9], in order to characterize the propagation of these waves in different conditions (tunnels, rural and urban scenarios, straight and curved routes). Although they are suited for communication between RRHs and MRs, mmWave frequencies might be best suited for an mMTC slice dedicated for transmission of train control and management system (TCMS) information between sensors and RRHs (both fixed along the track, such that LOS can be easily maintained) or between sensors inside the train measuring train parameters and the internal train communication network [7]. Sub-6Ghz bands can be used for longer range connectivity between MRs and macro BSs.

Next, we focus on an energy efficient approach using MRs to meet the QoS requirements of train passengers, while minimizing the energy consumption at UEs and the transmit power at MRs and BSs. Thus, it mainly targets the eMBB use case. However, the power savings at the BS and MRs can be diverted to support the operation of other slices corresponding to $\mathrm{mMTC}$ and/or URLLC as needed.

\section{PERFORMANCE ENHANCEMENT WITH MRS}

\section{AND INTELLIGENT RESOURCE ALLOCATION}

This section presents some results showing the enhanced performance due to the deployment of MRs on train wagons, coupled with intelligent resource allocation algorithms. The 
results show the enhanced performance obtained on both the indoor UE-MR links and the outdoor MR-BS links, in terms of QoS, modeled by having the UEs reach a given target rate, and in terms of energy consumption. The energy consumption at the BS and MRs is based on analyzing the transmit power, whereas at the UEs it is based on the total energy consumed by the circuitry of the mobile device, since the aim is to reduce the battery power consumed when accessing the network during high speed travel.

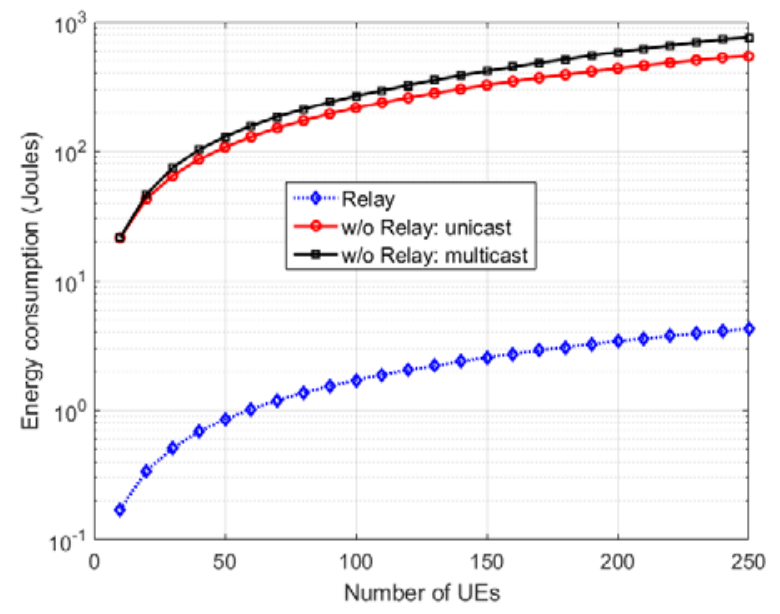

Fig. 2. Energy consumption of UEs in Joules versus the total number of UEs when each wagon is equipped with a MR, such that the MRs are connected to LTE BSs outside the train and using WiFi inside the train.

Fig. 2 shows the total energy consumption of UEs in a train with ten wagons, along with 25 UEs per wagon. The results shown correspond to receiving 1 Mbits per each UE. The scenarios with and without MRs are compared. The multicasting scenario corresponds to a situation where the UEs are receiving the same content from the BS, e.g., the live broadcast of a football match. When the wagons are equipped with a MR, it is connected to an LTE BS with a total bandwidth of $5 \mathrm{MHz}$, corresponding to 25 resource blocks (RBs). Each MR communicates with the LTE BS outside the train, and provides indoor WiFi connectivity to the UEs inside the train. Due to avoiding penetration loss and providing SR connectivity to UEs, the energy savings are large. For example, the total consumption for 200 MTs is around 3.5 Joules compared to 430 and 600 Joules for unicasting and multicasting without MRs, respectively.

It could be possible to use the same technology indoor and outdoor when MRs are deployed. In fact, LTE/LTE-A could be used inside the train wagons while the MRs can also be connected to the BS using LTE/LTE-A. This approach can be extended to 5G using the accessing schemes that will be adopted as enhancements to orthogonal frequency division multiple access (OFDMA). In such a scenario, the MR would act similarly to an indoor femtocell connecting the UEs inside each wagon to the network. Appropriate frequency subdivision of the various subcarriers should be performed between the indoor and outdoor portions of the network. This can be easily supported through carrier aggregation while benefiting from the fact that the indoor transmissions of each MR can be confined to its wagon to minimize interference.

Fig. 3 shows the performance of a resource allocation algorithm for allocating the LTE-A RBs from the MRs to the UEs inside the train and from the BSs to the MRs outside the train. It is assumed that there are 25 UEs per wagon, and that a bandwidth of $5 \mathrm{MHz}$, corresponding to $25 \mathrm{RBs}$, is available to each MR for indoor allocation. Another $10 \mathrm{MHz}$ is assumed dedicated for allocation between the BS and the MRs (corresponding to $50 \mathrm{RBs}$ ). One RB is assumed to be allocated per UE on the UE-MR link, and one RB is assumed allocated to each MR on the MR-BS link, at each transmission time interval (TTI). Thus, the problem becomes an assignment problem that is solved by the Hungarian algorithm. It is shown that with the algorithm proposed by the authors in [5], all UEs achieve their target data rate $\mathrm{R}^{\mathrm{U}}{ }_{\mathrm{T}}$ when the value of $\mathrm{R}_{\mathrm{T}}^{\mathrm{U}}$ is less than $3 \mathrm{Mbps}$, and more than 95\% achieve their target rate when $\mathrm{R}_{\mathrm{T}}^{\mathrm{U}}$ reaches 4 Mbps (assuming all UEs have the same target rate). The proposed algorithm performs the same RB assignment as in the Hungarian algorithm, and then performs an additional optimization on the transmit power to maximize the number of served UEs while minimizing the transmitted power at the MRs (additional details along with the list of simulation parameters can be found in [5]). The iterative algorithm is a lower complexity resource allocation algorithm proposed in [6] and based on equal power allocation. It also allows all UEs to achieve their target data rate when $\mathrm{R}_{\mathrm{T}}^{\mathrm{U}}$ is less than $3 \mathrm{Mbps}$, but around 80\% achieve their target rate when $\mathrm{R}_{\mathrm{T}}^{\mathrm{U}}$ reaches $4 \mathrm{Mbps}$. The power consumption due to indoor transmission by the MRs, although minimized by the proposed algorithm, is negligible compared to the power consumed at the BS, shown in Fig. 4. In fact, due to the presence of the indoor antennas of the MRs in each wagon, the distance between UEs and MRs is short, thus leading to efficient resource allocation with reduced power consumption.

Fig. 4 shows the results of the outdoor BS-MR link for a train with ten wagons, along with 25 UEs per wagon. The target rate per UE is set to $0.4 \mathrm{Mbps}$, such that the total rate requested by each $\mathrm{MR}$ from the $\mathrm{BS}$ is the aggregate of the target rates requested by the UEs in its served wagon. Thus, each MR would request a rate of $10 \mathrm{Mbps}$ from the BS. The same algorithms that were implemented for the UE-MR link are implemented for the MR-BS link, but with the aggregate rate used as target rate instead of the individual UE rate. The results are plotted versus the distance between the first wagon and the nearest BS. 


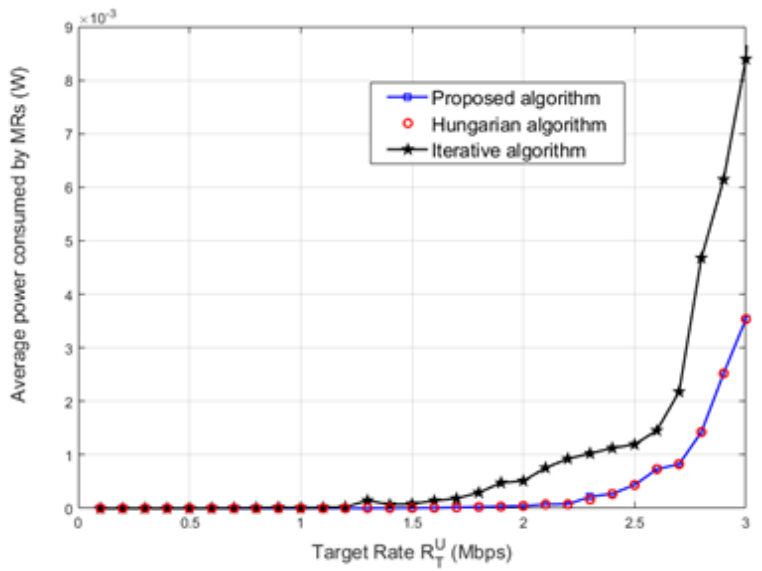

(a)

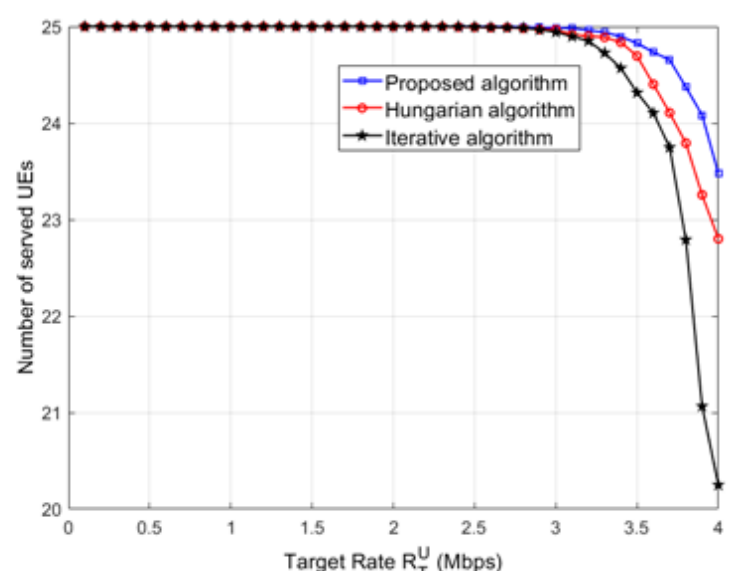

(b)

Fig. 3 Number of served UEs versus the target rate in the access UE-MR link inside the train.

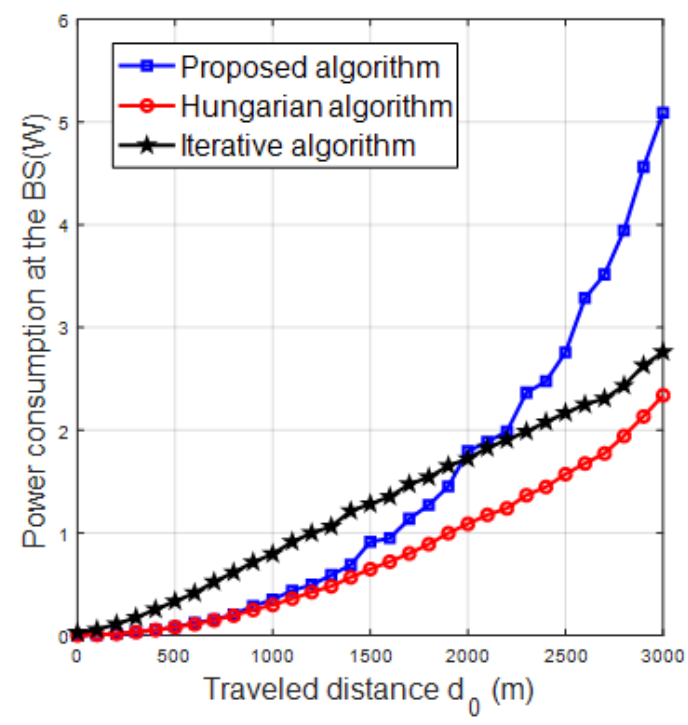

(a)

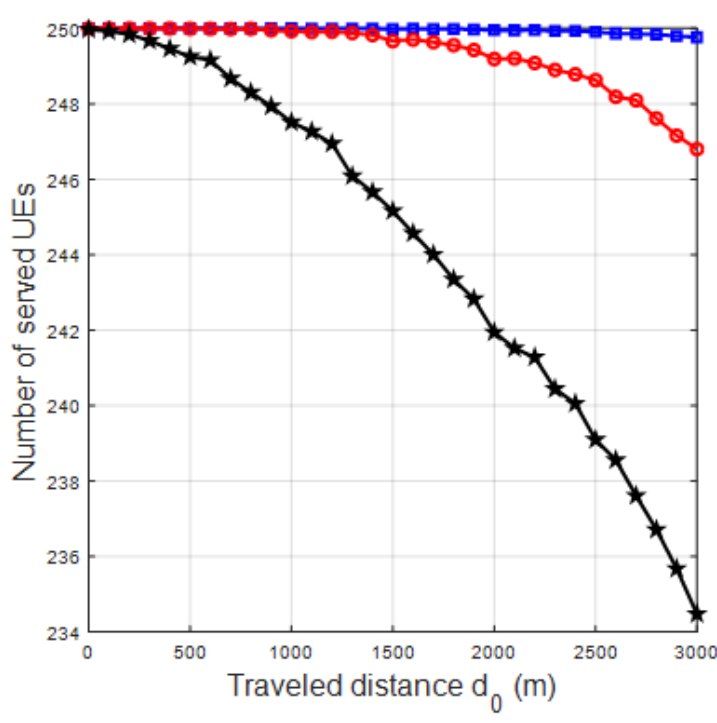

(b)

Fig. 4. Performance on the MR-BS link versus the distance between the $\mathrm{BS}$ and the train with a UE target rate of $\mathrm{R}_{\mathrm{T}}^{\mathrm{U}}=$ $0.4 \mathrm{Mbps}$.

Fig. 4 shows that all UEs can be served with the proposed algorithm, with the Hungarian and iterative algorithms serving around $98 \%$ and $94 \%$ of the UEs, respectively. The proposed algorithm leads to low transmit power consumption at the BS, comparable to that of the Hungarian algorithm, as long as the distance between the BS and the train is not too large. When the distance increases, the power consumption of the proposed algorithm increases judiciously as needed in order to successfully serve all UEs on the train. Having RRHs installed along the railroad would have reduced the perceived distance with the BS and led to reduced power consumption. Furthermore, dynamic beamforming with massive MIMO antennas at the BS would have served the same purpose. These network design tradeoffs would depend on the access rights to the railroad, safety regulations allowing the installation of additional infrastructure, and cost constraints [1].
It should be noted that the results without MRs (using the same algorithms) are dramatically worse, as shown in Fig. 5. For example, for a distance of $100 \mathrm{~m}$ with the BS, $100 \mathrm{UEs}$ can be successfully served for a target rate of $\mathrm{R}_{\mathrm{T}}^{\mathrm{U}}=0.5 \mathrm{Mbps}$. This number reduces to less than 20 UEs with the proposed approach when the distance reaches $2000 \mathrm{~m}$ (the Hungarian and iterative algorithms would serve less than five UEs in this case).

In addition, it should be noted that massive MIMO operation of MRs is not used in the presented results. Had the MRs been assumed to operate as a single 5G massive MIMO array under the control of a single BBU, along with the use of more bandwidth, then the data rate results would be orders of magnitude higher, since the MRs would be under a centralized control. The results corresponding to independent $\mathrm{MR}$ operation would thus constitute a lower bound on performance. 


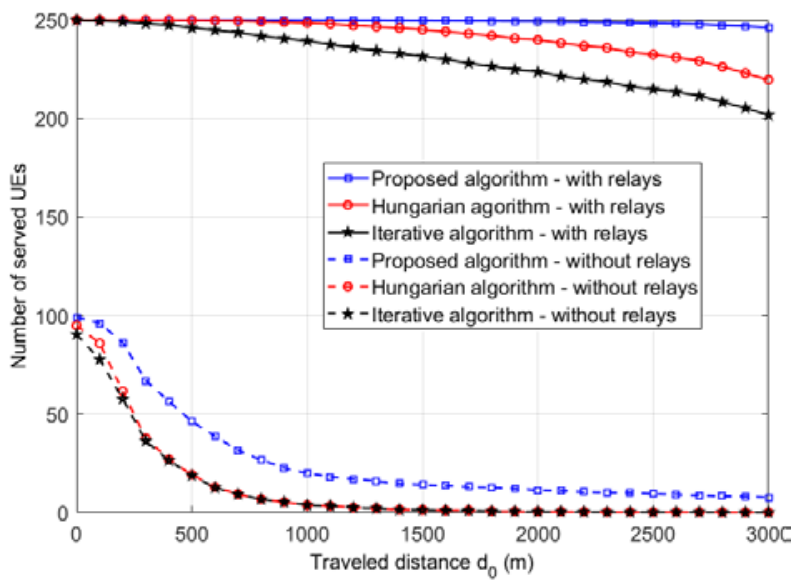

Fig. 5. Total number of served UEs versus the distance between the $\mathrm{BS}$ and the train with a UE target rate of $\mathrm{R}_{\mathrm{T}}^{\mathrm{U}}=$ $0.5 \mathrm{Mbps}$.

\section{Challenges AND Potential Solutions}

This section describes some important challenges faced by railway communications, and discusses potential solutions to address them.

\section{A. Doppler Effect}

Due to the high speed of the trains, Doppler effect cannot be neglected. In fact, it leads to high inter-carrier interference (ICI) in OFDMA-based systems. Doppler effect mitigation techniques are investigated in the literature, in addition to modified versions of OFDMA that lead to more robust behavior in face of the Doppler effect [10]. For example, the resource block filtered orthogonal frequency-division multiplexing (RB-F-OFDM) was shown in [10] to outperform other waveforms. The results of Figs. 2-5 are based on allocating one RB per device and thus are easily applicable with RB-F-OFDM, although both RB-F-OFDM and the resource allocation algorithms used in this paper can be easily adapted to accommodate more RBs per device. Another approach to deal with the Doppler effect is to benefit from the varying value allowed for subcarrier spacing (SPS) in 5G. In [8], it was shown that SPS greater than $60 \mathrm{KHz}$ would be more suitable than the $15 \mathrm{KHz}$ used in LTE/LTE-A. Although the results would differ, the same algorithms used to generate the results of Figs. 2-5 can be applicable independent of the SPS.

\section{B. Handover}

When the UEs inside a train are connected directly to the BS, the handover of a large number of devices at high speed becomes a significant challenge. This problem is significantly alleviated by using MRs at the top of the train, although not necessarily at the frequency of one MR per wagon. Since UEs are connected to MRs, the problem becomes that of handing over the MRs, i.e., a lower number of more powerful devices.

In fact, in [11], a concept similar to MRs is adopted where each train is equipped with two antennas: one at the front and the other at the rear. One antenna might perform a handover while the other is still connected to the initial BS in order to ensure a smooth transition to the users. Once the first handoff is successful, the second antenna can be handed over. A similar approach was adopted in [12], while taking into account the details of handover between macrocell BSs (used mainly for train control data), or between micro BSs (used for user traffic) under the coverage of the same macro BS. This approach is extended in [4] to a situation where multiple antennas (more than two) are available on the train top. With the first-in-first-out (FIFO) approach of [4], whenever an antenna succeeds in performing a handover from one small cell to another, then the other antennas communicate with it internally over the train's fiber network. The switching occurs between antennas depending on which antenna is first successfully handed over to the target BS.

Handover performance can be further enhanced by power adjustment as suggested in [13]. Under this approach, when the train enters the overlap area between the serving cell and target cell, the serving cell increases its transmit power in order to delay the handover (thus avoiding premature handovers) until the train becomes closer to the target cell. At that moment, the target cell starts increasing its power whereas the serving cell decreases its power so that the handover can smoothly take place.

\section{Channel State Information (CSI) Feedback}

To perform efficient resource allocation, the BS needs to have relatively accurate channel state information (CSI). With the MR approach, the UEs and the MRs are at relatively fixed positions with low mobility with respect to each other, which solves the problem of having UEs continuously report to the BS CSI information that will arrive outdated due to the high speed of the train. However, there is still a need for CSI exchange between the MRs and the BS. A potential solution is the use of the "predictor antenna" method, e.g., see [14]. Since the trajectory is well-known along the track, then, when several antennas are deployed on the train roof, a subsequent antenna will reach the position of the preceding antenna after a very short time period. The CSI sent by the first antenna will be irrelevant to that antenna when the BS makes and notifies the MR of its scheduling decision, but it will be highly correlated with the CSI of the subsequent antenna, since it would have reached the position of its preceding antenna. Thus, with this approach, a "dummy" antenna can be used to provide CSI for the first antenna element, and then the CSI of each antenna can be used for the antenna following it. This approach is feasible with 5G, especially with the adoption of massive MIMO techniques involving the use of large numbers of antennas. Each MR can possess a multitude of antenna elements placed on the roof of its wagon (e.g., patch antennas can be easily embedded within the structure of the wagon) that can be used with the predictor antenna method. Another method could be to use a "cross-slice" approach to avoid CSI feedback. In fact, the train position and speed are known 
accurately via feedback of GPS information in addition to sensor data from the rail track and onboard the train. This data communication is part of the mMTC slice, but if it is available at the BS, e.g., via edge caching techniques, then this information could be used for beamforming without the need for CSI. Indeed, the MRs with their antennas positioned outside the train will have a LOS link most of the time with the BSs deployed along the track (in tunnels RRHs can compensate for the lack of LOS). Thus, "geometrically" knowing the position of the train and its speed can allow the BS to perform beam steering with highly directive beams directed towards the MRs to boost the BS-MR signal (especially for the eMBB or URLLC slices), without traditional CSI feedback. Recent studies have already used the train position and speed information for beam switching [15].

\section{Energy Efficient Operation}

Energy efficient operation of the railway communications networks is often overlooked in the literature, where the focus is on topics of higher priority in these networks. However, careful planning of the network, in conjunction with intelligent resource allocation, can still lead to energy efficiency while maintaining safety and critical network operation. For example, small cells along the track dedicated purely to eMBB can be put into sleep mode when there are no trains passing. The energy efficient methods described in this paper (results of Figs. 2-5) can lead to energy savings that can be directed to other slices when needed (e.g., URLLC). In addition, even if all BSs are active, reduced power consumption due to intelligent resource allocation can lead to cost savings for the operators on the long run.

\section{E. Public Safety}

In addition to mission critical transmissions form railroad monitoring sensors in case of emergency, video surveillance transmission is an important factor for maintaining security in trains. Surveillance cameras deployed in train wagons have to send their monitoring videos to a control center. This imposes a significant load on the network. However, with the advances in video coding, e.g. through the use of High Efficiency Video Coding (HEVC), video can be coded in a scalable way with varying data rates. For example, by varying the video coding parameters, the data rate can be made to vary between $64 \mathrm{kbps}$ and 2Mbps. Thus, the high quality videos can be locally processed on a dedicated server in the train using artificial intelligence/machine learning techniques to detect any problems, e.g., by analyzing the behavior of passengers from the recorded videos and detecting normal/abnormal situations. In parallel, these videos can be transmitted to the control center at low data rates with "reasonable" quality in normal situations. Whenever an emergency is detected, the transmitted video quality can be increased along with an alert message to the control center. Appropriate resources should be reserved in the wireless network to maintain a "basic" operation of this network. However, whenever an emergency arises, resources can be diverted from the slice dedicated to passenger transmissions as needed to provide the utmost priority for the safety network.

\section{F. Security}

The data transmitted by the sensors that are part of the railroad management and control network should be secured against malicious attacks. Due to the nature of these sensors, it might be difficult to implement advanced cryptographic techniques. Therefore, physical layer security methods and/or lightweight encryption techniques are a suitable solution to address this problem while adapting to the constraints of the sensing devices. Nevertheless, more advanced cryptographic techniques can be implemented to secure the video surveillance data discussed in the previous subsection, in order to protect the privacy of the passengers and the integrity of the data sent to the public safety control center. In addition, physical layer security methods could be used to combat eavesdropping (thus maintaining privacy) or jamming (aiming to prevent the data from reaching the control center).

\section{G. Laws and Regulations}

Implementing a state-of-the-art communication network for railroad communications is not only dependent on pure technical aspects. In addition to determining whether a single network should be used for passenger communications and rail control/management or two separate networks, other details should be addressed. For example, it has to be decided if the network deployed will be under the control of a mobile network operator, of a dedicated service provider, of the train operating company, etc. If it is not under the control of the railroad authorities, appropriate rights and permissions should be granted to allow the communications service provider or network operator to access the properties of the rail network and install the needed infrastructure [1]. Furthermore, the equipment installed should take into account the rail safety standards and not hinder the operation of the trains. Thus, careful coordination and smart decision making should take place to avoid any bureaucratic hurdles that might affect the efficient deployment and operation of the railroad communication network.

\section{ConCLUSIONS}

In this paper, connectivity and energy efficiency in modern $5 \mathrm{G}$ railways were discussed. The use of mobile relays in conjunction with intelligent resource allocation was analyzed in terms of energy efficiency and enhanced connectivity. Relevant challenges to train connectivity were also presented, and suitable solutions that would be interesting for additional research investigations were outlined.

\section{REFERENCES}

[1] S. Friedner, R. Womersley, and T. Treacher, "Connected train and customer communications: Rail and digital industry roadmap", Tech. Rep., Rail Safety and Standards Board (UK), January 2018.

[2] L. Yan, X. Fang, H. Li, and C. Li, "An mmWave wireless communication and radar detection integrated network for 
railways", IEEE 83rd Vehicular Technology Conference (VTC Spring), May 2016.

[3] J. Kim et al., "A comprehensive study on mmWave-based mobile hotspot network system for high-speed train communications", IEEE Transactions on Vehicular Technology, Doi: 10.1109/TVT.2018.2865700 (early access), August 2018.

[4] S. Banerjee, S. M. Rakshit, M. Hempel, and H. Sharif, "5GUCDA in high speed rail with multi antenna-to-logical cell circular FIFO mapping strategy", International Conference on Computing, Networking and Communications (ICNC), March 2018.

[5] H. Ghazzai, T. Bouchoucha, A. Alsharoa, E. Yaacoub, M. S. Alouini, and T. Al-Naffouri, "Transmit power minimization and base station planning for high-speed trains with multiple moving relays in OFDMA systems”, IEEE Transactions on Vehicular Technology, Vol. 66, No. 1, pp. 175-187, January 2017.

[6] E. Yaacoub, R. Atat, A. Alsharoa, and M.-S. Alouini, "Mobile relays for enhanced broadband connectivity in high speed train systems”, Physical Communication (Elsevier), vol. 12, pp. 105115, September 2014.

[7] A. Gonzalez-Plaza et al., "5G communications in high speed and metropolitan railways", 11th European Conference on Antennas and Propagation (EUCAP), March 2017.

[8] F. Hasegawa et al., "High-speed train communications standardization in 3GPP 5G NR", IEEE Communications Standards Magazine, vol. 2, no. 1, pp. 44-52, March 2018.

[9] D. He et al., "Channel measurement, simulation, and analysis for high-speed railway communications in 5G millimeter-wave band", IEEE Transactions on Intelligent Transportation Systems, vol. 19, no. 10, pp. 3144-3158, October 2018.

[10] Q. Zheng et al., "Comparison of 5G waveform candidates in high speed scenario", URSI General Assembly and Scientific Symposium (GASS), August 2017.

[11] S. Xie, X. Yu, and Yuan Luo, "A seamless dual-link handover scheme with optimized threshold for $\mathrm{C} / \mathrm{U}$ plane network in highspeed rail", IEEE 83rd Vehicular Technology Conference (VTC Spring), May 2016.

[12] J. Zhao, Y. Liu, Y. Gong, C. Wang, and L. Fan, "A dual-link soft handover scheme for $\mathrm{C} / \mathrm{U}$ plane split network in high-speed railway", IEEE Access, vol. 6, pp. 12473-12482, Jan. 2018.

[13] Y. Lu, K. Xiong, P. Fan, Z. Zhong, and B. Ai, "The effect of power adjustment on handover in high-speed railway communication networks", IEEE Access, vol. 5, pp. 2623726250, November 2017.

[14] D.-T. Phan-Huy, M. Sternad, and T. Svensson, "Making 5G adaptive antennas work for very fast moving vehicles," IEEE Intelligent Transportation Systems Magazine, vol. 7, no. 2, pp. 71-84, Summer 2015.

[15] Y. Cui, X. Fang, Y. Fang, and M. Xiao, "Optimal nonuniform steady mmWave beamforming for high-speed railway", IEEE Transactions on Vehicular Technology, vol. 67, no. 5, pp. 43504358, May 2018.

\section{BIOGRAPHIES}

Elias Yaacoub (S'08, M'10, SM'14) received the B.E. degree in Electrical Engineering from the Lebanese University in 2002, the M.E. degree in Computer and Communications Engineering from the American University of Beirut (AUB) in 2005, and the PhD degree in Electrical and Computer Engineering from AUB in 2010. Between 2010 and 2014, he worked as a Research Scientist/R\&D Expert at the Qatar Mobility Innovations Center
(QMIC). Afterwards, he joined Strategic Decisions Group (SDG) where he worked as a Consultant till February 2016. Then he joined the Arab Open University (AOU) an Associate Professor. Since February 2018, he is a freelance researcher/consultant; he is also affiliated with AUB as a Research Associate and part-time faculty member. His research interests include Wireless Communications, Antenna Theory, IoT, and Physical Layer Security.

Ahmad Alsharoa (S'14, M'18) was born in Irbid, Jordan. He received the B.Sc degree from Jordan University of Science and Technology (JUST), Irbid, Jordan, in January 2011 and the M.Sc. degree from King Abdullah University of Science and Technology (KAUST), Thuwal, Saudi Arabia in May 2013, both in Electrical Engineering. He received the PhD degree with comajors in Electrical Engineering and Computer Engineering from Iowa State University (ISU), Ames, Iowa, USA, in May 2017. He was a postdoctoral research associate University of Central Florida (UCF), Orlando, Florida, USA, from September 2017August 2018 before joining Virginia State University as Assistant Professor in August 2018. His current research interests include: Computer security, Wireless networks, mmWave communications, internet of things (IoT), cooperative relay networks, and MIMO communications, among others.

Hakim Ghazzai (M'15) was born in Tunisia. He is currently a Research Scientist at the Stevens Institute of Technology, NJ, USA. He received his PhD degree in Electrical Engineering from King Abdullah University of Science and Technology (KAUST) in Saudi Arabia. He received his Diplome d'Ingenieur and Master of Science degree in telecommunication engineering with highest distinction from the Ecole Superieure des Communications de Tunis (SUP'COM), Tunisia, in 2010 and 2011, respectively. Before joining Stevens, he worked as a Research Scientist at the Qatar Mobility Innovations Center (QMIC), Qatar, from 2015 to 2017 and as a Visiting Researcher in Karlstad University, Sweden in the Spring of 2018. His general research interests include mobile and wireless networks, green communications, internet of things, and UAV-based communications.

Mohamed-Slim Alouini (S'94, M'98, SM'03, F'09) was born in Tunis, Tunisia. He received the Ph.D. degree in Electrical Engineering from the California Institute of Technology (Caltech), Pasadena, CA, USA, in 1998. He served as a faculty member in the University of Minnesota, Minneapolis, MN, USA, then in the Texas A\&M University at Qatar, Education City, Doha, Qatar before joining King Abdullah University of Science and Technology (KAUST), Thuwal, Makkah Province, Saudi Arabia as a Professor of Electrical Engineering in 2009. At KAUST, Prof. Alouini leads the Communication Theory Lab (ctl.kaust.edu.sa) and his current research interests include the modeling, design, and performance analysis of wireless communication systems. 